\title{
Delayed Perforation Occurring after Endoscopic Submucosal Dissection for Early Gastric Cancer
}

\author{
Soo Hoon Kang, Kyungho Lee, Hyun Woo Lee, Ga Eun Park, Yun Soo Hong and Byung-Hoon Min \\ Department of Medicine, Samsung Medical Center, Sungkyunkwan University School of Medicine, Seoul, Korea
}

\begin{abstract}
Delayed perforation is a very rare complication of endoscopic submucosal dissection (ESD), with a reported incidence of $0.1 \%$ to $0.45 \%$. Few reports exist on the clinical features and outcomes of delayed perforation after ESD, and it is unclear whether the optimal management strategy is emergency surgery or endoscopic closure with conservative treatment. Here, we report two cases of delayed perforation occurring after ESD for early gastric cancer. In both cases, lesions were located in the antrum, and tumor depths were confined to the mucosal layer. Total procedure times for ESD were 25 and 45 minutes, respectively. Because delayed perforation may be associated with excessive thermal damage and necrosis of the muscle layer, treatment with emergency surgery should be used instead of conservative management in cases of delayed perforation after ESD.
\end{abstract}

Key Words: Coagulation; Endoscopy; Perforation; Stomach neoplasms; Surgery

\section{INTRODUCTION}

Endoscopic submucosal dissection (ESD) is widely used as one of the main therapeutic modalities for early gastric cancer (EGC) because of its minimal invasiveness, high rate of en bloc resection, and comparable long-term outcomes to surgery. ${ }^{1-3}$ One of the major complications of ESD is perforation during the procedure, which has a reported incidence of 3.5\% to $7 \% .{ }^{4}$ In most cases, perforation occurring during ESD can be successfully treated by immediate endoscopic closure and conservative management. Delayed perforation is defined as a perforation occurring after ESD, without a visible gastric wall defect during ESD and without free air on chest radiography performed immediately after ESD. Delayed perforation is very rare, with a highest reported incidence of $0.45 \%{ }^{5}$ Because it is very rare, few reports exist on the clinical features and outcomes of delayed perforation after ESD. In addition, it is unclear whether the optimal management strategy for

Received: May 21, 2014 Revised: July 10, 2014

Accepted: August 11, 2014

Correspondence: Byung-Hoon Min

Department of Medicine, Samsung Medical Center, Sungkyunkwan University School of Medicine, 81 Irwon-ro, Gangnam-gu, Seoul 135-710, Korea

Tel: +82-2-3410-3409, Fax: +82-2-3410-6983, E-mail: bhmin@skku.edu

(®) This is an Open Access article distributed under the terms of the Creative Commons Attribution Non-Commercial License (http://creativecommons.org/ licenses/by-nc/3.0) which permits unrestricted non-commercial use, distribution, and reproduction in any medium, provided the original work is properly cited. delayed perforation should be emergency surgery or endoscopic closure with conservative treatment. Here, we report two cases of delayed perforation occurring after ESD for EGC.

\section{CASE REPORTS}

\section{ESD procedure}

The ESD procedures were performed as previously described. ${ }^{1}$ After the tumor margins were delineated by chromoendoscopy with indigo carmine spray, marking dots were placed around the lesion. Then, normal saline mixed with epinephrine, indigo carmine, and glycerol was injected into the submucosal layer to separate the lesion from the muscle layer. After making a submucosal cushion, a circumferential precutting and then submucosal dissection were performed with a dual knife (KD-650L; Olympus, Tokyo, Japan) and insulatedtip (IT)-2 knife (KD-611L; Olympus). VIO 300D was used as the electrosurgical unit (ERBE, Tuebingen, Germany). Minor bleeding during submucosal dissection was controlled using the electrosurgical knives in a swift coagulation mode. Bleeding from large vessels during precutting or submucosal dissection, and visible vessels in the ESD-induced artificial ulcer after dissection, were coagulated using a hemostatic forceps (FD-410LR; Olympus) in a soft coagulation mode at $80 \mathrm{~W}$ current. 


\section{Case 1}

An 86-year-old man was referred to our hospital for the treatment of EGC. A tumor was located on the anterior wall of the antrum. Endoscopically estimated tumor size was $1.2 \mathrm{~cm}$ (Fig. 1A). Significant perigastric lymphadenopathy and distant metastasis were not found on abdominal computed to-
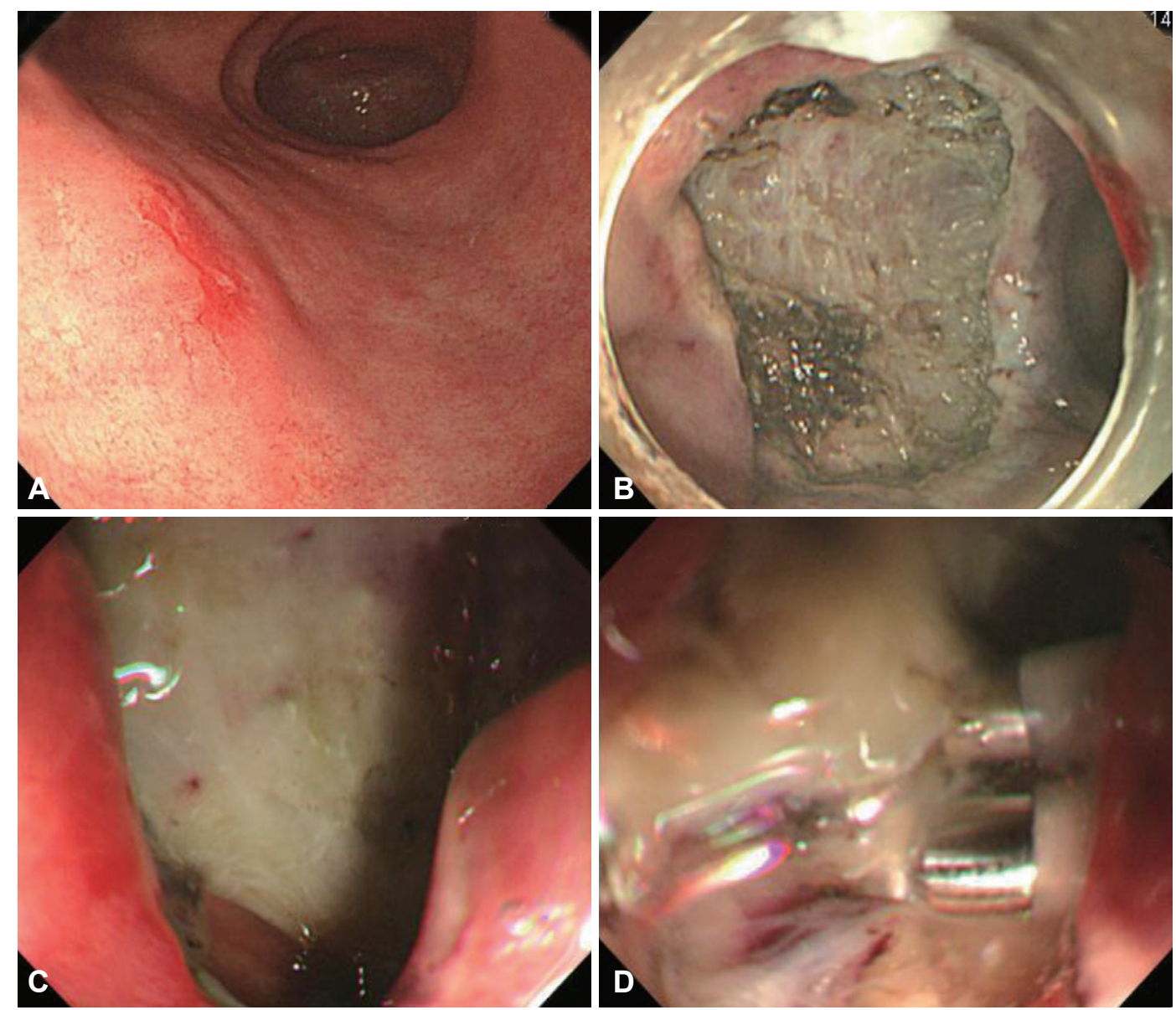

Fig. 1. (A) White light endoscopy image shows a $1.2 \mathrm{~cm}$-sized type Ilc early gastric cancer lesion on the anterior wall of the proximal antrum. (B) Appearance of the iatrogenic ulcer after endoscopic submucosal dissection (ESD). (C) Endoscopic view shows a $1 \mathrm{~cm}-s i z e d$ gastric wall defect in the ESD-induced ulcer base. (D) Perforation hole is successfully closed with three endoclips.
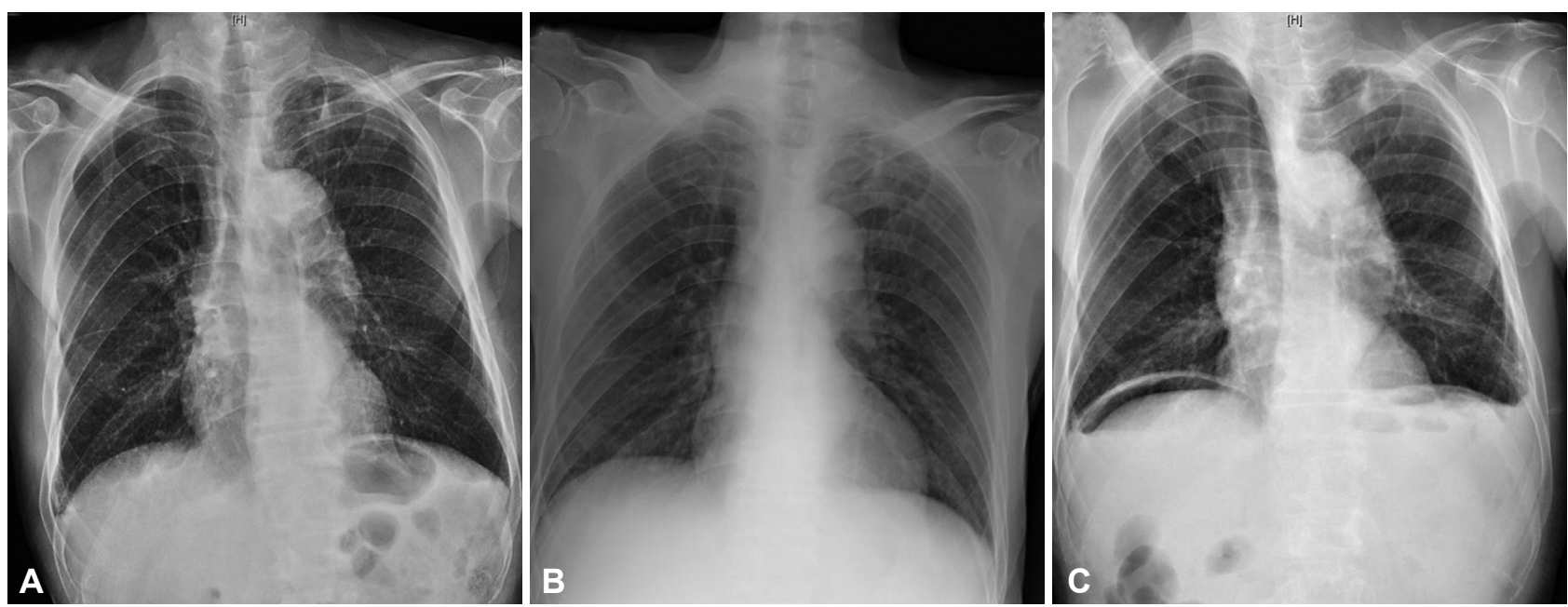

Fig. 2. (A) Chest X-ray taken immediately after the endoscopic submucosal dissection (ESD) procedure reveals no free air. (B) No free air is seen on chest radiography taken 12 hours after the ESD procedure, when the patient first complained of abdominal pain. (C) Follow-up chest radiography at 23 hours after the ESD procedure shows free air. 
mography (CT). ESD was successfully performed in en bloc fashion, and all resection margins were tumor-free (Fig. 1B). Resected specimen size was $4.2 \mathrm{~cm}$. Total procedure time was 25 minutes. No free air was observed on chest radiography

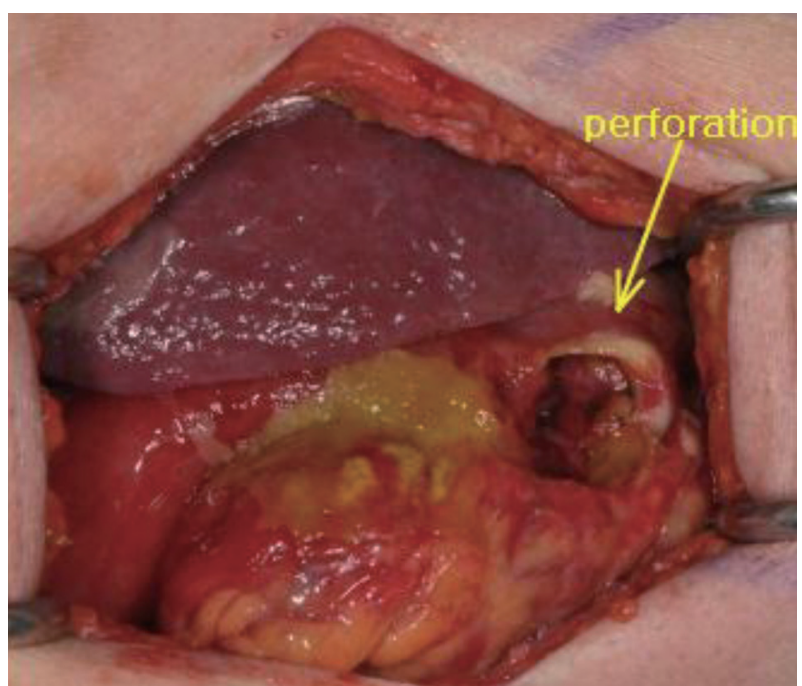

Fig. 3. A $3 \mathrm{~cm}$-sized laceration is found at the previous endoscopic submucosal dissection site, and primary open repair is performed. performed immediately after ESD (Fig. 2A). Pathological analysis of the ESD specimen indicated that the lesion was a $0.8 \mathrm{~cm}$-sized moderately differentiated adenocarcinoma confined within the lamina propria layer. No lymphovascular invasion was detected. Twelve hours after the ESD procedure, the patient complained of severe abdominal pain. Because he did not have fever and a follow-up chest radiography at that time revealed no free air (Fig. 2B), the patient was conservatively managed with a proton pump inhibitor and pethidine. Twenty-three hours after ESD, his pain aggravated, and a follow-up chest radiography showed free air (Fig. 2C). Emergency upper endoscopy revealed a $1 \mathrm{~cm}$-sized gastric wall defect in the ESD-induced ulcer base (Fig. 1C), and the perforation site was then closed with three clips (Fig. 1D). Because clipping seemed successful, the patient was conservatively managed with antibiotics, a proton pump inhibitor, and parenteral nutrition. With this treatment, his abdominal pain improved, and defecation occurred 3 and 4 days after clipping. Five days after clipping, the abdominal pain again exacerbated. Abdominal CT showed a large pneumoperitoneum complicated with ascites, suggestive of panperitonitis, and the patient agreed to undergo emergency explorative laparotomy. However, the
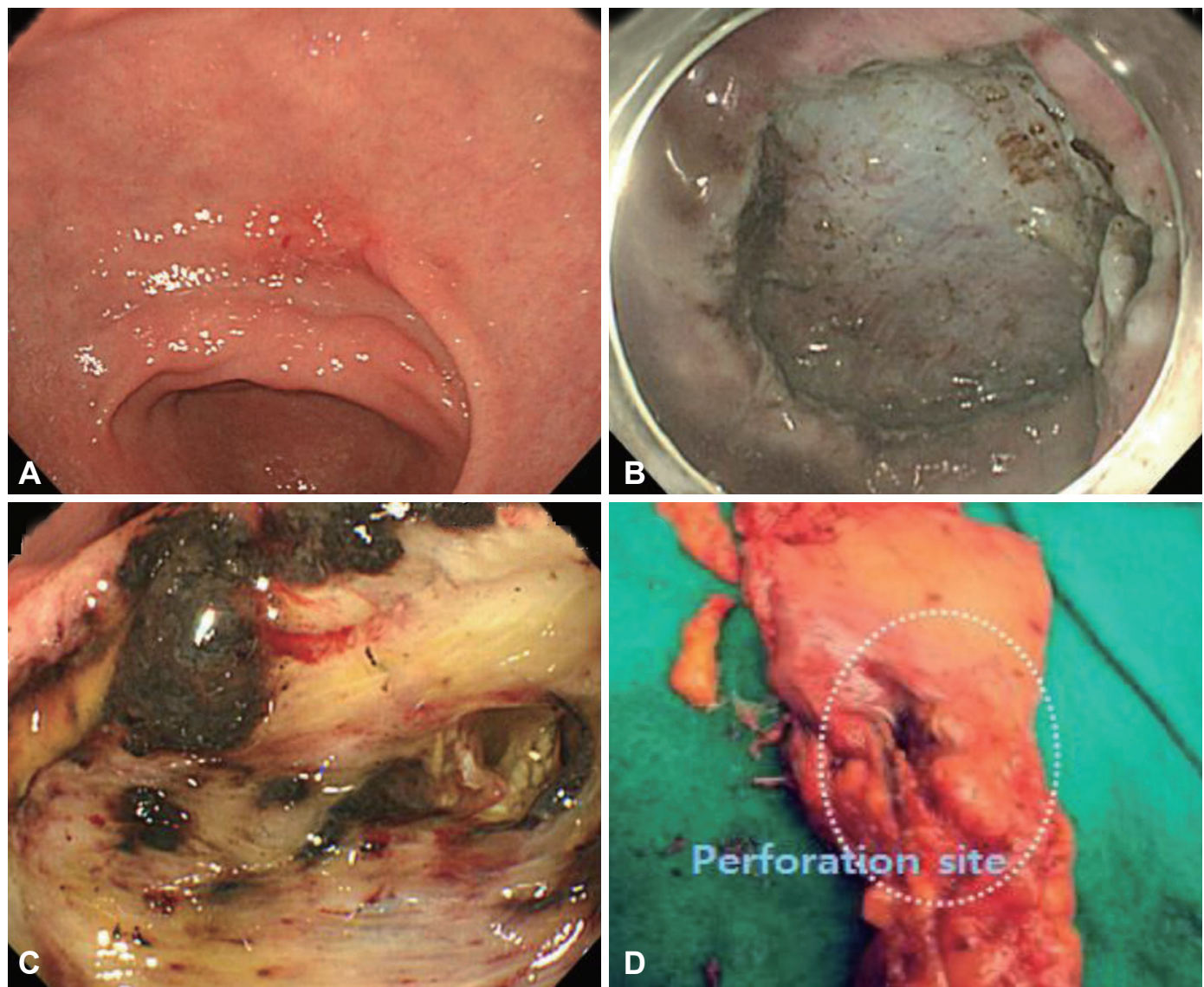

Fig. 4. (A) White light endoscopy image shows a $1.8 \mathrm{~cm}$-sized early gastric cancer on the lesser curvature of the antrum. (B) Appearance of the iatrogenic ulcer after endoscopic submucosal dissection (ESD). (C) Endoscopic view shows a $2 \mathrm{~cm}$-sized perforation in the ESD-induced ulcer base. (D) Pathologic specimen of the segmental resection shows a $2 \mathrm{~cm}$-sized perforation on the lesser curvature of the antrum. 
laparoscopic approach was not feasible in this patient due to massive complicated fluids and inflammatory tissues in the peritoneal space. During open surgery, a $3 \mathrm{~cm}$-sized laceration was found at the previous ESD site, and primary suturing of the laceration was performed without gastrectomy (Fig. 3). The patient was discharged 14 days after surgery without any further complication.

\section{Case 2}

A 67-year-old man underwent upper endoscopy as part of a routine check-up. He was diagnosed with a $1.8 \mathrm{~cm}$-sized EGC on the lesser curvature of the antrum (Fig. 4A). No significant enlargement of lymph nodes was detected on a CT scan of the abdomen. ESD was successfully performed in en bloc fashion, and all resection margins were tumor-free (Fig. 4B). Resected specimen size was $4.5 \mathrm{~cm}$. Total procedure time was 45 minutes. No free air was observed on a chest radiography taken immediately after ESD. Pathological review of the ESD specimen showed that the lesion was a $1.8 \mathrm{~cm}$-sized, welldifferentiated adenocarcinoma confined to the muscularis mucosa layer. No lymphovascular invasion was detected. Ten hours after the ESD, the patient complained of severe abdominal pain. A chest radiography taken at that time did not reveal free air, and the patient was afebrile. Despite conservative management with a proton pump inhibitor and pethidine, the patient's pain was aggravated, and a follow-up chest radiography taken at 15 hours after ESD showed free air. Emergency upper endoscopy revealed a $2 \mathrm{~cm}$-sized perforation in the ESD-induced ulcer base (Fig. 4C). Because the perforation was too large to attempt endoscopic clipping, the patient underwent emergent laparoscopic segmental resection (Fig. 4D). The patient was discharged 7 days after surgery without any complication.

\section{DISCUSSION}

Delayed perforation is a very rare complication of ESD, with a reported incidence of $0.1 \%$ to $0.45 \%{ }^{5,6}$ In our institution, one of the tertiary hospitals in Korea with the most experience performing ESD for EGC, a total of 3,432 patients underwent ESD between 2000 and 2013. Among them, only two cases of delayed perforation occurred $(0.06 \%)$. Owing to the low incidence of delayed perforation, few reports exist on its clinical features and outcomes. Hanaoka et al. ${ }^{5}$ described six cases of delayed perforation occurring after ESD for EGC. In their case series, the majority of the lesions were located in the upper third of the stomach and total procedure time was relatively long, ranging from 1.5 to 9 hours. Four of the six lesions needed deep submucosal dissection because of massive submucosal invasion and ulcer scarring. Therefore, the essential mechanism of delayed perforation after ESD was suggested to be electrical cautery during submucosal dissection or repeated coagulation that caused ischemic change to the gastric wall, resulting in necrosis. ${ }^{5}$ However, our case series demonstrated different features. Both EGC lesions were located in the antrum, and tumor depths were confined to the mucosal layer. Total procedure times for ESD were 25 minutes and 45 minutes, respectively. These features were similar to those reported by Ikezawa et al. ${ }^{6}$ In their case report, the EGC lesion was located on the greater curvature of the antrum and tumor depth was confined within the mucosal layer. The ESD procedure took only 25 minutes, and the size of the perforation was only $3 \mathrm{~mm}$. On the basis of these findings, Ikezawa et al. ${ }^{6}$ suggested that shrinkage or disappearance of vessels penetrating the gastric wall, caused by electrical cautery, might have contributed to the occurrence of delayed perforation. As the number of reported cases is limited, further studies are required to identify the mechanism and risk factors for delayed perforation after ESD.

Because of limited experience, it is unclear whether the optimal management strategy for delayed perforation after ESD is emergency surgery or endoscopic closure with conservative treatment. In the case series of Hanaoka et al., ${ }^{5}$ all but one patient underwent surgical treatment. Among the five patients treated surgically, three underwent omentoplasty without gastrectomy. All surgically treated patients were discharged early without any operation-related complications. In our case series, conservative management was attempted in the first case after successful endoscopic closure of the perforation. However, on the 5th day after the clipping, panperitonitis developed. A laparoscopic approach was not possible owing to complicated inflammation, and the patient eventually underwent open surgery. This patient was discharged 14 days after surgery. In contrast to the first patient, the second patient underwent emergency surgery immediately after the occurrence of delayed perforation was detected. Laparoscopic segmental resection without gastrectomy was successfully performed, and the patient was discharged 7 days after surgery.

Delayed perforation may be caused by blunt damage from ischemic change rather than sharp laceration by an electrosurgical knife, and the size of the necrotic area and perforation can be large. This makes endoscopic closure with clips difficult and can result in late dehiscence even after successful clipping. As in our first case, late surgery can be complicated by severe inflammation, which inevitably leads to open surgery. In cases of early surgery, however, delayed perforation can be successfully treated by laparoscopic simple suture or segmental resection without radical gastrectomy. Given these points, emergency surgery may be preferable to endoscopic closure with conservative treatment in cases of delayed perforation after ESD. 


\section{Conflicts of Interest}

The authors have no financial conflicts of interest.

\section{REFERENCES}

1. Gotoda T, Yamamoto H, Soetikno RM. Endoscopic submucosal dissection of early gastric cancer. J Gastroenterol 2006;41:929-942.

2. Lee JH, Hong SJ, Jang JY, Kim SE, Seol SY. Outcome after endoscopic submucosal dissection for early gastric cancer in Korea. World J Gastroenterol 2011;17:3591-3595.

3. Oka S, Tanaka S, Kaneko I, et al. Advantage of endoscopic submucosal dissection compared with EMR for early gastric cancer. Gastrointest
Endosc 2006;64:877-883.

4. Minami S, Gotoda T, Ono H, Oda I, Hamanaka H. Complete endoscopic closure of gastric perforation induced by endoscopic resection of early gastric cancer using endoclips can prevent surgery (with video). Gastrointest Endosc 2006;63:596-601.

5. Hanaoka N, Uedo N, Ishihara R, et al. Clinical features and outcomes of delayed perforation after endoscopic submucosal dissection for early gastric cancer. Endoscopy 2010;42:1112-1115.

6. Ikezawa K, Michida T, Iwahashi K, et al. Delayed perforation occurring after endoscopic submucosal dissection for early gastric cancer. Gastric Cancer 2012;15:111-114. 